\title{
Equivalent Nonlinearities for Studying Nonlinear Effects on Sampled OFDM Signals
}

\author{
João Guerreiro, Student Member, IEEE, Rui Dinis, Senior Member, IEEE, and Paulo Montezuma
}

\begin{abstract}
In this work we consider nonlinear effects on sampled orthogonal frequency division multiplexing (OFDM) signals and we present a method for obtaining a polynomial function with small degree that can be used to substitute severe nonlinear characteristics (possibly non-differentiable, as clipping functions), since it leads to signals with essentially the same spectral characteristics. This equivalent nonlinearity is then employed for performance evaluation of clipped OFDM signals, leading to accurate results with both conventional and optimum receivers.
\end{abstract}

Index Terms-Gaussian signals, intermodulation analysis, nonlinear effects, OFDM.

\section{INTRODUCTION}

$\mathbf{I}$ $\mathrm{T}$ is well known that OFDM signals [1] have high peak-toaverage power ratio (PAPR), which leads to implementation difficulties, namely at the amplification level. Over the last two decades, several techniques were proposed to reduce the PAPR of OFDM signals (see [2] and references within). Among them, the simplest and more flexible involve clipping operations [3][5]. However, the clipping is a nonlinear operation that leads to both in-band distortion and out-of-band radiation, which must be taken into account when evaluating the performance of OFDM schemes.

The Gaussian-like nature of OFDM signals with large number of subcarriers can be used for the statistical characterization of nonlinearly distorted OFDM signals [5], [6]. The basic approach is to decompose the signal at the output of the nonlinear device as the sum of two uncorrelated terms: a useful one, proportional to the original OFDM signal, and a distortion term [7], whose spectral characterization can be done based on classical intermodulation product analysis (IMP) analysis [5], [6].

This approach is relatively straightforward and can be employed for most memoryless nonlinearities. However, the number of required IMPs is related to the degree of the polynomial

Manuscript received November 15, 2014; accepted February 8, 2015. Date of publication February 12, 2015; date of current version April 8, 2015. This work was supported in part by Fundação para a Ciência e Tecnologia under the projects UID/EEA/50008/2013 (plurianual funding and project HETNET), EnAcoMIMOCo EXPL/EEI-TEL/2408/2013, CoPWIN PTDC/EEI-TEL/1417/ 2012 and grant SFRH/BD/90997/2012. The associate editor coordinating the review of this paper and approving it for publication was $\mathrm{Q}$. Du.

J. Guerreiro is with the Faculdade de Ciências e Tecnologia (FCT), 2829-516 Monte de Caparica, Portugal.

R. Dinis is with Instituto de Telecomunicações, 1049-001 Lisboa, Portugal, and Faculdade de Ciências e Tecnologia (FCT), 2829-516 Monte de Caparica, Portugal.

P. Montezuma is with UNINOVA, Monte de Caparica, Portugal and Faculdade de Ciências e Tecnologia (FCT), 2829-516 Monte de Caparica, Portugal.

Digital Object Identifier 10.1109/LCOMM.2015.2403326 approximation of the nonlinear characteristic, which means that for strong nonlinear effects and/or non-smooth nonlinear characteristics (such as the ones inherent to clipping) the required number of IMP to accurately characterize the distortion term can be very high.

In this work we consider nonlinear effects on sampled OFDM signals. We propose a simple method to obtain a polynomial function with low degree whose output has the same spectral characteristics as the nonlinearly distorted signal, for a given nonlinearity and oversampling factor. This equivalent polynomial nonlinearity is then employed to evaluate the impact of clipping effects on sampled OFDM signals. We obtain very accurate estimates of the power spectral density (PSD) of the output signals, as well as the corresponding signal-tointerference ratio (SIR) levels. We also use it to obtain the performance of optimum receivers for clipped OFDM signals.

\section{Clipped OFDM Signals}

Let us consider an OFDM signal that carries $N$ complex data symbols. These symbols are represented by $\mathbf{S}^{T x}=$ $\left[S_{0}^{T x} S_{1}^{T x} \ldots S_{N-1}^{T x}\right]^{T} \in \mathbb{C}^{N}$, where $\mathbb{E}\left[\left|S_{k}^{T x}\right|^{2}\right]=2 \sigma_{S}^{2}$ (we considered normalized quadrature phase shift keying (QPSK) constellations with $\left.S_{k}^{T x}= \pm \sigma_{S} \pm j \sigma_{S}\right)$. We also consider the addition of $(M-1) N$ idle subcarriers at the edges of the useful band, so that the corresponding time-domain samples represent a sampled OFDM signal with an oversampling factor of $M$. At the inverse discrete Fourier transform (IDFT) input we have the vector $\mathbf{S}=\left[S_{0} S_{1} \ldots S_{N M-1}\right]^{T} \in \mathbb{C}^{N M}$. At the IDFT output we have the time-domain samples $\mathbf{s}=$ $\mathbf{F}^{-1} \mathbf{S}=\left[\begin{array}{lll}s_{0} s_{1} & \ldots & s_{N M-1}\end{array}\right]^{T} \in \mathbb{C}^{N M}$, with $\mathbf{F}$ denoting the $N M$-point discrete Fourier transform (DFT) matrix. When $N$ is large, the time-domain samples $\mathbf{s}=\left[\begin{array}{lll}s_{0} s_{1} & \ldots s_{N M-1}\end{array}\right]^{T} \in$ $\mathbb{C}^{N M}$ have Gaussian nature, i.e., $s_{n} \sim \mathcal{C N}\left(0, \sigma^{2}\right)$, where $\sigma^{2}=$ $\sigma_{S}^{2} /\left(N M^{2}\right)$ denotes the variance of the real and imaginary parts of each sample. Consequently, the corresponding absolute values $\mathbf{r}=|\mathbf{s}|=\left[r_{0} r_{1} \ldots r_{N M-1}\right]^{T} \in \mathbb{C}^{N M}$ have a Rayleigh distribution with variance $\sigma^{2}$. The time-domain sample $s_{n}=$ $r_{n} \exp \left(j \theta_{n}\right)$ (where $\theta_{n}$ is the original phase) is distorted by a bandpass memoryless leading to $y_{n}=f\left(r_{n}\right) \exp \left(j \theta_{n}\right)$, where $f\left(r_{n}\right)=f\left(\left|s_{n}\right|\right)=A\left(r_{n}\right) \exp \left(j \Theta\left(r_{n}\right)\right)$, with $A\left(r_{n}\right)$ denoting the amplitude modulation-amplitude modulation (AMAM) conversion function and $\Theta\left(r_{n}\right)$ denoting the amplitude modulation-phase modulation (AM-PM) conversion function. Although our approach can be applied to any bandpass memoryless nonlinearity, in this work we focus on an ideal envelope clipping with normalized clipping level $s_{M} / \sigma$ (the extension to other cases is straightforward), that has a zero AM-PM characteristic. From the Gaussian nature of OFDM signals, the $n$-th nonlinearity output sample $y_{n}$ can also be decomposed in two uncorrelated terms (this is a consequence of Bussgang's 